\title{
A biotechnological approach to microbial based perfumes and flavours
}

\begin{abstract}
Flavours and fragrances represent over a quarter of the world market and most of the flavouring and perfume compounds are produced through chemical synthesis or by extraction from natural materials like plant and animal sources. Fragrances and flavours finds application in food, feed, cosmetic, chemical and pharmaceutical industries. Plant and animal sources are an important source of bioflavours but these bioactive compounds are present in minor quantities thus making their isolation and formulation very expensive. The other bio-route for flavour synthesis is based on de novo microbial processes (fermentation) or on bioconversions of natural precursors using microbial cells or enzymes (biocatalysis). This mini review is an attempt to focus on the potential of microorganisms to be used as small biofactories for the production of bio-flavours and fragrances.
\end{abstract}

Volume 2 Issue I - 2015

\author{
Sneh Gupta, ${ }^{2}$ Charu Gupta, 'Amar P Garg, ${ }^{3}$ \\ Dhan Prakash' \\ 'Amity Institute for Herbal Research and Studies, Amity \\ University, India \\ ${ }^{2}$ Department of Zoology, R.G.P.G. College, India \\ ${ }^{3}$ Department of Microbiology, CGS University, India
}

Correspondence: Charu Gupta, Amity Institute for Herbal Research and Studies, Amity University, Uttar Pradesh, Noida-201302, India, Tel 919958905744 ,

Email charumicro@gmail.com

Received: July 22, 2014 | Published: January 07, 2015

Keywords: microorganisms, fragrances, bio-flavours, food additive

\section{Introduction}

Flavours and fragrances are generally extracted from plants and animals since times immemorial. The structure of flavour compounds from plant sources range from single to complex substances. Their chemical structures are elucidated by the modern sophisticated techniques like mass spectroscopy and NMR that is followed by their chemical synthesis. Flavours are generally used as food additives and they are mostly produced through artificial means i.e. chemical synthesis or by extraction from plant and animal sources. Flavours and fragrances are widely used in food, beverage, feed, cosmetic, detergent, chemical and pharmaceutical formulations. Recently due to consumer's increased interest and health awareness in natural products, there has been more stress towards the use of natural fragrances and flavours obtained from natural sources. Research has shown that microbes can also be used to produce aromas and fragrances. Man is using microbial systems to impart new aromas to the fermentation products like beer, wine, cheese etc from ages. Vanillin (1874) and coumarin (1868) were the first synthetic fragrance and flavour compounds made available for use in the food industry. The worldwide demand of flavours and fragrances was estimated to be US\$ 16 billion in the year 2013. Most of these flavouring and fragrance compounds are prepared by chemical technology and only a small fraction of the demand are met from plant or through microbial sources. ${ }^{l}$

The natural ingredients are added to formulations for efficiency, emolliency or emotional appeal. The chemical synthesis of fragrances is not desirable as this is not ecofriendly. Moreover it lacks substrate selectivity and specificity, which normally causes the formation of undesirable racemic mixtures as side products. This reduces the process efficiency and increases downstream costs. The direct extraction of natural flavours from the plants is also difficult. The plant raw materials contain very low amounts of desired compounds, thus making the extraction process quite expensive and time consuming. Besides their production is dependent on various factors like climate, temperature that are difficult to control and they are more susceptible to plant diseases. Due to the above mentioned drawbacks and the consumers increasing interest in natural products, have directed research for other suitable alternative strategies for producing the natural flavours and fragrance compounds. ${ }^{2}$ But sometimes these natural flavours are found only in certain specific exotic plant species. An attractive alternative method for flavour and fragrance synthesis is based on de novo microbial processes (fermentation) or on bioconversions of natural precursors using microbial cells or enzymes (biocatalysis). ${ }^{2}$ Microorganisms are well known for the production of flavor and aroma chemicals for the past 60 years. The characteristic flavor of any compound is mainly due to the presence of many volatile and nonvolatile components with diverse chemical and physicochemical properties. These compounds are generally present within the complex matrices. Research has proved that the nonvolatile compounds contribute mainly to the taste, while the volatile ones determine the taste and aroma of a particular compound. There are numerous compounds that are responsible for the aroma of the food products, such as aldehydes, esters, alcohols, dicarbonyls, short to medium-chain free fatty acids, lactones, methyl ketones, phenolic compounds and sulphur compounds. ${ }^{3}$ These flavour compounds can be produced naturally by using microbial cultures or their enzyme preparations although plant cell cultures can also be used. There are many microorganisms that naturally synthesize flavours as the secondary metabolites during fermentation. More recently as per Chemical and Engineering news, genetically engineered bacteria and yeast are being developed that carry the genes for producing the fragrance from plants.

The first report of using genetically engineering strains of bacteria became prevalent in year 2010 when there was an acute shortage of fragrance oil commonly extracted from Pogostemon cablin (Patchouli). The patchouli plant yields an essential oil that is used as a fragrance in incense sticks and other personal and health care products. It was reported that rainy weather in Indonesia destroyed the medicinal shrub resulting in a poor harvest of fragrant oil. The 
condition further worsened when volcanic eruptions and earthquakes exacerbated supply problems (Chemical \& Engineering News). To meet the growing demands of the fragrance oil, genetically modified microbes and yeast were specifically designed to produce fragrances of patchouli plant. In addition other fragrances of bitter orange, grapefruit, rose and sandalwood that are otherwise hard to obtain naturally were also produced using genetically modified microbial strains. There are some biotech companies like Allylix, Isobionics, Givaudan, Firmenich, Evolva and International Flavors \& Fragrances that are involved in producing the microbial based perfumes and flavours from abundant sugar feed-stocks by fermenting sugars. ${ }^{I}$ The first product generated from microbe is 'valencene', a citrus molecule that is usually found in the peel of Valencia oranges and the other is 'nootkatone' that is present in grapefruit peel. These compounds are commonly used in fruit-flavored drinks and perfumes. Vanillin scent that is normally produced through synthetic reactions can also be now produced by microbial fermentation. The advantage of producing perfumes from microbes is that they are more ecofriendly and natural. Plant sources are generally unreliable: they are often susceptible to natural disasters and plant diseases that lead to supply shortages. The engineered bacteria can produce the fragrance in much larger quantities without harvesting the rare plant (Wired Science). Flavor and fragrance molecules produced during microbial fermentation promises an abundant supply that is independent of climate conditions and natural disasters with uniform quality of the product. ${ }^{4}$ It is generally estimated that $95 \%$ of chemicals used in fragrances are derived from petroleum with artificial flavouring worth $\$ 20$ billion industry. Nature produces scents from a class of compounds called esters that are produced by reacting an alcohol with an organic acid. The ester isoamyl acetate smells like banana, methyl salicylate smells like winter green. Living cells like yeasts also produce esters that flavour beer and wine. Bacteria also produce esters that flavour cheeses. A group of researchers introduced the scent selective genes from yeast and introduced them into $E$. coli. The enzymes were tweaked to alter the biochemical pathways in the cells so as to manipulate the ester that was produced. This novel technique has been patented and researchers used metabolic engineering to make E. coli produce a synthetic banana smell and nicknamed it "Eau d'coli". 4

\section{Market demand of microbial based perfumes and fragrances}

The biotechnology provides an attractive alternative for producing fragrant and flavour molecules in large quantities. This has paved the way for the biotechnological firms like DSM, a Dutch-based multinational life-sciences and materials-sciences company (in full Koninklijke DSM N.V., or Royal DSM) and another largest chemical producer company headquartered in Germany BASF (Badische Anilin- und Soda-Fabrik). These companies have invested a lump sum amount of $\$ 13.5$ million towards the microbial based production of flavour and fragrance molecules in collaboration with a company named Allylix that is based in San Diego.

Both the companies Allylix and Isobionics are producing two citrus molecules valencene from the peel of the Valencia orange and nootkatone from grapefruit peels but it can also be produced from valencene. Both the flavour molecules are used in fruit-flavored beverages and perfumery industry. They also find application in personal care and cleaning products. Another consulting firm on flavour and fragrance Leffingwell \& Associates reported that the overall industry that produced synthetic molecules from petroleum feed- stocks was worth $\$ 21.8$ billion in 2011. Microbial based fermentation process has the ability to synthesize high-demand molecules such as valencene and nootkatone that can significantly alter supply dynamics (Lux Research, 2014). The report suggested that there is potential for biosynthetic routes to completely replace any natural sources of flavour and fragrance molecules. The pharmaceutical and biotech companies often work in partnerships and adopt most secretive policies and conditions to develop these natural molecules. ${ }^{5}$

Thus with the help of biotechnological tools, one can produce flavour and fragrance molecules more cheaply and in larger quantities that was not possible by using either chemical synthesis or extraction from plants. Metabolically engineered microbes can produce a variety of natural molecules ranging from patchoulol, linalool, nerolidol, valencene to sclareol through fermentation. These molecules are more environment-friendly and are natural, e.g. a biotech company Givaudan has patented a microbial biosynthetic pathway for the production of vanillin. Vanillin is a common flavoring agent that can be now cheaply produced from phenol using microbes instead of costly natural vanilla. Similarly, biotech companies will also produce other natural molecules that are only available in small quantities from botanical sources, ${ }^{5}$ for example, citrus peel extracts are already used in large quantities, but there is huge demand for more essential oils. Another flavour molecule like esters can also be produced through genetically engineered bacteria. Ester molecules are responsible for many sensory tastes and are the backbone of any flavour and fragrance industry. Its market demand grosses $\$ 20$ billion annually. The researchers introduced genes from yeast into $E$. coli bacteria and manipulated the Acyl-CoA pathway. The genetically modified microbial cells utilize an enzyme called O-acetyltransferases to make esters from acyl-Coenzyme A (acyl-CoA) molecules. The scientists were able to synthesize a complete ester by turning on certain potential metabolic pathways and shutting down others, and thus they can virtually hand-pick the type of molecule the bacteria produce.

Based on the above study, a patent application was also filed suggesting the details of making terpenes through microbial fermentation. Generally Escherichia coli or yeast such as Saccharomyces cerevisiae is engineered for isoprenoid production by insertion of one or two genetic pathways used by plants to make terpene precursors. The microbe also gets genes coding for an enzyme 'terpene synthase' that makes a particular terpene from one or more precursors. The resulting terpene can be functionalized through hydroxylation, isomerization, oxidation, reduction or acylation. The major advantage of the microbial production is that even a rare flavour or fragrance can be isolated and produced in large quantities from inaccessible place. This helps in reaching a scale where the natural molecule can be used in common consumer products. Biotechnology can thus help to replace natural perfume and flavor ingredients from plants such as lavender, jasmine, or ylang-ylang and it can be further enhanced by the addition of fruit peels, roots, grasses, evergreen needles, woods, resins, and balsams to produce molecules like esters, ethers, aldehydes, hydrocarbons and ketones through fermentation. More than 75,000 terpenes have been identified in nature that can be utilized by scientists to synthesize the bio-flavours through metabolic engineering of microbial biosynthetic pathways. Thus large volumes of complex chiral compounds can be produced quite economically. ${ }^{6}$

Research has also shown that the starting material for fragrance is farnescene, a type of sesquiterpene that is derived by microbial fermentation from petroleum feedstocks. The fermentation route 
allows leapfrogging certain steps that are normally involved in the chemical production, thus reducing cost and environmental benefits. Another biotech Swiss firm 'Evolva' has identified pathway for the fermentation production of saffron components that is the monoterpene glycoside picrocrocin. It would be commercially available from year 2015 or 2016. In addition, Evolva has two ongoing flavor ingredient collaborations with International Flavors \& Fragrances, the thirdlargest company in the industry. Evolva is also working on unraveling the biosynthetic pathways of vanillin. It has also made key components of the plant-derived sweetener Stevia through fermentation in yeast Vanillin is the most consumed flavour that is commonly extracted from tropical Vanilla orchids (Vanilla planifolia). However, it meets only less than $1 \%$ of the world market demand. The cost of production of synthetic vanillin is much cheaper (>US\$15/kg) as compared to natural plant product (US\$1200-4000/kg). ${ }^{1}$

A biotech firm 'Allylix' develops the terpene molecules and creates their own formulations as finished products. ${ }^{7}$ Terpenes are made up of multiple isoprene units and depending on the no. of isoprene units can be further classified as monoterpenes (contain two isoprenes), sesquiterpenes (contain three isoprene units) such as valencene and nootkatone. Recently a third sesquiterpene called epivone is added that is structurally related to $\beta$-vetivone, one of the key components of vetiver oil, an essential oil obtained from roots of Chrysopogon zizanioides with a rich, woody aroma. Another biotech company Isobionics, based in the Netherlands, has a business model similar to Allylix and also produces terpenes. Its recent product is the sesquiterpene $\beta$-elemene. Today, $\beta$-elemene is obtained through a costly extraction from ginger root. Although $\beta$-elemene has flavor and fragrance applications, but its main therapeutic interest is as a potential anticancer drug. Another biochemical based fuel firm Amyris is also in close partnership with the two leading flavor and fragrance firms Givaudan and Firmenich that provide a competitive advantage. Both companies work for fragrance ingredients in two phases. First is a co-development phase where they develop a bio-based product. The second is the commercialization phase in which the flavor and fragrance house uses the natural molecules in their products. These companies were also working on fermentation-derived version of patchouli. The fragrance of the oil is due to the presence of terpenes like patchoulol and nor-patchoulenol. ${ }^{6}$

Thus biotechnology through metabolic engineering of bacteria aims to develop the natural flavours and fragrances in all seasons that are unaffected by weather conditions including monsoon, drought, or even by volcano. ${ }^{1}$

\section{Flavours and fragrances: types and synthesis}

Flavours and fragrances are broadly divided into two categoriesnatural and nature-identical. Natural flavours are prepared by extraction from plants or by enzymatic or microbial processes, and 'nature-identical' flavours and fragrances are synthesized chemically or by conversions of natural substrates. The 'natural' flavours are produced either by de novo synthesis in microbes or plants or through single-step biotransformation of natural substrates by microbes or their enzymes or plant cells (e.g. synthesis of nootkatone using citrus cell cultures). In de novo synthesis, microbes transform carbon or nitrogen compounds into flavour molecules with the help of enzymes such as lipases, esterases, proteases, nucleases and some glycosidases. These enzymes are extensively used in industry for the synthesis of flavouring compounds as they catalyze singlestep 2 transformations of substrates into natural flavour molecules. Furthermore, microbiological or enzyme based processes have also been developed where complex substrates such as lignin, phenylpropanoids and phenolic stilbenes are converted to the desired flavours and fragrances. ${ }^{8}$

\section{Types of natural and nature-identical flavours}

Natural flavours are those flavouring substances or preparations which are obtained by appropriate physical, enzymatic or microbiological processes and obtained from vegetal or animal origin material. Generally speaking, natural flavour includes essential oil, oleoresin, essence or extractive, protein hydrolysate, distillate of any product of roasting, poultry, eggs, dairy products or fermentation products. According to Code of Federal Regulations, USA (1990), the term 'natural flavour' includes the essential oil, oleoresin, essence or extractive, protein hydrolysate, distillate of any product of roasting, heating or enzymolysis, which contains the flavouring constituents derived from a spice, fruit juice, vegetable or vegetable juice, edible yeast, herb, bud, bark, root, leaf or similar plant material, meat, seafood, poultry, eggs, dairy products or fermentation products thereof, whose significant function in food is imparting flavouring rather than nutrition. According to EC Flavour Directive (88/388/EEC) natural flavours are defined as flavouring substances or preparations which are obtained by appropriate physical, enzymatic or microbiological processes from material of plant or animal origin. Both definitions state that natural flavours include products obtained through microbial or enzymatic processes as long as the precursor/raw material are natural and obtained through physical or bio-processes and that the precursor and product can be found in nature or are part of traditional foods. The chief physical processes for obtaining natural flavours are extraction, distillation, concentration, crystallization; from animal sources (beef, chicken, seafood) or plant sources (spices, mushroom, citrus, fruits, mints). 'Nature-identical' flavours are those products that occur in nature but are produced through a chemical (a non-natural) process. This mode of production is no longer accepted as consumer-friendly. ${ }^{9}$ There are some aroma compounds e.g. esters (ethyl and butyl acetates, ethyl butyrate, caproate, isobutyrate, isovalerate, 2-methyl butyrate, menthyl acetate), aldehydes and ketones, (acetaldehyde, diacetyl), acids (acetic, butyric, caproic, caprylic, isobutyric, isovaleric, 2-methyl butyric) and lactones (gamma-decalactone) which can either be produced by microbial fermentation or by using enzymes (Table 1). ${ }^{10}$

Table I Flavours and fragrances produced by some microorganisms or using enzymes adopted from Krings \& Berger. ${ }^{10}$

\begin{tabular}{lll} 
Flavour type & Flavour active component & Microorganism/enzyme involved in production \\
\hline Almond & Benzaldehyde & Ischnoderma benzoinum (a bracket fungus) \\
Apple and pine apple & Butyric acid & Clostridium butyricum \\
Flavour component of dairy products & 2,3 -butanedione & Lactic streptococci \\
Rose-like odor & Citronellal & Rhodotorula minuta (yeast) \\
Flavour component of many essential oils & $(+)$-Curcumene & Saccharomyces cerevisiae (baker's yeast)
\end{tabular}


Table Continued....

\begin{tabular}{lll}
\hline Flavour type & Flavour active component & Microorganism/enzyme involved in production \\
\hline Peach & $\gamma$-Decalactone & Yarrowia lipolytica (yeast) \\
Coconut-peach & $\delta$-Decalactone & Enzymatic reduction of the $\alpha, \beta$-unsaturated compound (massoia lactone) \\
Flavour component of many essential oils & $(+)$-Dehydro-curcumene & Saccharomyces cerevisiae (yeast) \\
Citrus type fragrance & $(-)$ lsopulegol & Lipase (Pseudomonas sp.) \\
Mint & $(-)$-Menthol & Lipase (Candida rugosa) \\
Expensive fragrance compound & Nor-patchoulenol & Pithomyces sp. (mould) \\
Flavour component of many essential oils & $(+)$-Nuciferal & Saccharomyces cerevisiae (yeast) \\
Rosary & Phenolethanol & Kluyveromyces sp. (yeast) \\
Spearmint flavour & $\beta$-pinene & Lipase \\
Raspberry & Raspberry Ketone & Beauveria bassiana (fungus) \\
Chocolate flavours & Thaumatin and monellin & Kluyveromyces sp. (yeast) \\
Flavour component of many essential oils & $(+)$-Turmerone & Saccharomyces cerevisiae (yeast) \\
Vanilla & Vanillin & Pycnoporus cinnabarinus (fungus) \\
\hline
\end{tabular}

Bacterial colonization in roots \& fragrance oil composition

Scientific studies have shown that the fragrance oil obtained from a tropical grass like Vetiver is dependent on the composition of microbial communities present in and around the roots of the plant. The composition of vetiver root oil (commonly used as perfume) depends on the type of bacteria found living in the plant. Thus by altering the make-up of bacterial communities the composition of oil can be manipulated. The scientists further investigated the role of bacteria in the creation of oil and the ability of different bacterial strains found in the roots of plant to grow using Vetiver oil as the sole carbon and energy source. They found that a number of members of Enterobacteriaceae strains are very successful. The researchers also investigated the method of alteration of oil's structure by these microbes. It was found that each of the microorganisms metabolized the raw vetiver oil and produced some new compounds that are not present in the raw oil but are found in commercially available oils. ${ }^{11}$ The scientists also compared the behaviour of axenic vetiver i.e. Vetiver with no other organism or microbe present and found that only very small number of aroma producing compounds (sesquiterpenoids) are produced. However when the main sesquiterpenoid (beta-caryophyllene) was externally fed to the bacteria, other aroma producing compounds were also produced. Thus the bacterial communities living in the root directly affects the composition of oil and the aroma producing compounds that would otherwise not be present.

\section{Perfume oil production by microbes from aromatic grasses (Vetiver)}

The essential oil of vetiver is used in the cosmetic and perfumery industries. Recent studies have shown that group of bacteria resides in the root of a tropical grass Vetiver. The Vetiver root cells produce few oil precursors, which in turn are metabolized by the root bacteria to increase the complexity of Vetiver oil. The bacteria are present both in oil-producing cells as well as in root locations that are closely associated with the essential oil. These bacteria promote the production of essential oils, and also change the molecular structure of the oil, giving it different flavours and properties like termicidal, insecticidal, antimicrobial and antioxidant. The bacteria responsible for this transformation include high $\mathrm{G}+\mathrm{C}$ Gram-positive bacteria, alpha-, beta- and gamma-proteobacteria and the microbes belonging to the Fibrobacteres/ Acidobacteria group. The Vetiver grass is the only grass cultivated specifically for its root essential oil, which is made up of chemicals called sesquiterpenes. These are used in plants as pheromones and juvenile hormones. The essential oils also contain alcohols and hydrocarbons, which, together with the sesquiterpenes are primarily used in perfumery and cosmetics. The perfumery and flavouring industry could benefit from the increased variety that these bacteria provide to the smell and taste of these oils. This research opens new avenues in the biotech arena of natural bioactive compounds and pharmaceutical, perfumery and flavouring industries may exploit the selected microbial strains and widen their metabolic libraries. ${ }^{12}$ This is a good example depicting ecological role of plantmicrobial associations where the metabolic interplay between a plant (with few simple molecules) interact with root bacteria that in turn bio-transform them into a variety of bioactive compounds. ${ }^{11}$

Moreover, the content of oil produced also depends on geographical conditions. The studies reported that the properties of Vetiver oil change significantly and depends on the environment in which it was grown. This opens up the possibility of using these bacterial colonies to directly manipulate the oil composition. This can be done either by in vitro method using specific bacterial strains to convert the raw material into the desired composition or secondly by in vivo method by manipulating the bacterial colonization of the plant root. ${ }^{11}$ It is well known that soil around a plant is enriched in chemicals that are exuded by its roots, e.g. the roots of young maize plant exude large quantities of chemicals called benzoxazinoids (BXs) that play an important role in plant defence against pests above the ground in its stem and leaves. Scientists have found that a number of bacterial genes are responsible for this association with BX chemicals, thereby encouraging Psudomonas putida to migrate towards the plant. Studies have shown that the presence of Psudomonas putida accelerated the breakdown of BX molecules suggesting that the bacteria have evolved the ability to detoxify the root environment by using BX molecules as an energy source. This study has opened up new avenues for further research. One interesting lead came from the analysis of the bacterial genes that were switched on in the presence of root-produced BX chemicals. This analysis suggested that the BX chemicals not only recruit the bacteria to the root surface, but they also activate processes in these bacteria that can help to suppress soil-borne diseases. This is important as the plant is not only recruiting beneficial microbes but also regulating their behavior. The next line of research would 
be to obtain a molecular blueprint of the microbial communities that are shaped by these root chemicals and to investigate the beneficial impacts of these microbes on plant growth, health and soil quality. ${ }^{13}$

Another study has shown that maize crops emit certain specific chemical signals which attract growth-promoting microbes to live amongst their roots. This is the first chemical signal that has been shown to attract beneficial bacteria to the maize root environment This research could be particularly useful in protection of our crops by fighting against soil-borne pests and diseases. Disease resistant varieties of plants can be developed by breeding plants that are able to suppress disease and promote the plant growth promoting rhizobacteria (PGPR). With this, scientists hope to minimize the use of fertilizers and pesticides in agriculture. ${ }^{13}$ It is believed that certain plants secrete extracellular chemicals from their roots that attract other organisms near the root surface (rhizosphere). The microbial population around a plant's roots can be up to 100 times denser than elsewhere in the soil. The plant roots secrete simple soluble compounds such as sugars and organic acids that attract these microorganisms as they are a good source of energy. However complex chemicals are not known to serve as attractants because they are considered to be toxic. Certain common soil bacterium such as Pseudomonas putida uses these chemical toxins to locate a plant's roots. The plant benefits from the presence of these bacteria because they increase the availability of otherwise insoluble plant nutrients like iron and phosphorus and make them available. Furthermore, they also help by competing against harmful bacteria around the root system thus providing protection from the soil borne pathogens.

\section{Flavors and fragrance chemicals}

Alcohols: Alcohols (ethanol) are produced as a result of bacterial fermentations, various yeast also produces long-chain complex alcohols that possess unique organoleptic properties. In a study, Saccharomyces cerevisiae was immobilized on delignified cellulosic material and gluten pellets. The delignified cellulosic material resulted in higher amounts of ester production while gluten pellets produced higher amounts of alcohol. ${ }^{14}$ An important aroma-related alcohol is 2-phenylethanol which possesses a rose-like smell. It is usually chemically synthesized from substrates such as toluene, benzene, styrene, or methylphenylacetate. ${ }^{15}$ The natural 2-phenylethanol is mainly extracted from rose petals that involve a high-cost process. Specific strains of yeast like Kluyveromyces marxianus, Saccharomyces cerevisiae, Hansenula anomala are also capable of producing aroma compounds by bioconversion of 2-phenylalanine into 2-phenylethanol. ${ }^{16}$ However, there is a limitation of synergistic inhibition due to the presence of ethanol and 2-phenyl ethanol that reduces the tolerance of Saccharomyces cerevisiae in conversion to 2-phenylethanol. ${ }^{17}$

Methyl ketones: Methyl ketones contribute to cheese flavors and hence are in great demand. The presence of methyl ketones $\left(\mathrm{RCOCH}_{3}\right)$ was first observed in the spores of mold ripened cheese Penicillium roqueforti. ${ }^{2}$ The characteristic odour and taste of ripened cheese is due to the presence of methyl ketones, particularly methyl n-pentyl ketone as well as other short chain that contribute to fruity-spicy notes to fragrances. The mechanism of action of $P$. roqueforti is that the fungal mycelium converts fatty acid chains with less than 14 carbon atoms $\left(\mathrm{RCH}_{2} \mathrm{CH}_{2} \mathrm{CO}_{2} \mathrm{H}\right)$ into methyl ketones. In each reaction, the acids are oxidized to methyl ketones with one less carbon atom than the original acid. Similarly it is also possible to convert vegetable oil and triglycerides to methyl ketones. ${ }^{2}$
Diacety Diacetyl: Diacety Diacetyl $\left(\mathrm{CH}_{3} \mathrm{COCOCH}_{3}\right)$ is a naturally occurring flavour chemical that possesses a strong typical 'butter' odour and flavour on dilution. Therefore it is widely used to mimic the buttery taste to the dairy and other milk products. Diacetyl are also used in perfumes and in reconstituting essential oils. Diacetyl is closely related to acetoin. Acetoin is frequently found with diacetyl but contributes little or no flavor by itself. It was assumed that diacetyl was produced from acetoin by microbiological oxidation. The major diacetyl producing bacteria are Lactococcus lactis, Lactobacillus $\mathrm{sp}$, Streptococcus thermophilus, and Leuconostoc mesenteroides. A method for increasing the diacetyl production from bacteria such as $S$. diactilactis, S. cremoris and S. lactis has been patented. The use of humectants such as glycerol or sucrose lowers the water activity of the medium and results in greater diacetyl production. The production of diacetyl is further enhanced by a low $\mathrm{pH}$ (less than 5.5), low temperature and aeration. A pH below 5.5 enhances the activity of citric acid permease and reduces the activity of diacetyl reductase. ${ }^{2}$

Lactones: Lactones are chemically defined as cyclic esters of $\gamma$-and $\delta$-hydroxy acids. Lactones are responsible for various taste and odour like oily-peachy, creamy, fruity, nutty, coconut, etc. Various fungi are capable of producing lactones from substrates like triolein, sebum, lecithin, oleic acid and Tween 80. A coconut aroma is very popular as a food flavour and chemically they include $\gamma$-octalactone and $\gamma$-nonalactone. Another lactone having a coconut odour is 6-pentyl-2pyrone that is produced by fungus Trichoderma viridae. Other fungi involved include Tyromyces sambuceus and Cladosporium suaveolens that efficiently generate the coconut-flavoured lactones g-decalactone and d-dodecalactone from substrates ricinoleic acid and linoleic acid, respectively. The milky, buttery and coconut-like flavour provided by these lactones are desirable in dairy and milk products. However, lactones are also responsible for stale flavour of heated milk if used in excess amounts. Some yeasts such as Candida tropicalis or Yarrowia lipolytica possess the ability to degrade ricinoleic acid to C16, C14 and $\mathrm{C} 12$ acids and accumulate d-decalactone which contributes to fruity and oily flavour that are commonly used in peach, apricot and strawberry aromas. ${ }^{2}$

Butyric Acid: Butyric acid $\left(\mathrm{CH}_{3} \mathrm{CH}_{2} \mathrm{CH}_{2} \mathrm{CO}_{2} \mathrm{H}\right)$ is mainly produced by obligate anaerobic bacteria belonging to the genera Clostridium, Butyri-vibrio, Eubacterium and Fusarium. They are generally used in low concentration to supply butter-like flavour to different food products like natural cheese flavors. Its derivative pentyl butyrate provides a strong, ethereal, fruity, pungent odor resembling that of pear, pineapple and banana. Butyric acid is naturally present in ester form in butter ranging from $2-4 \%$ but its isolation process is very difficult and expensive. Thus microbial production through fermentation process is an attractive and cost effective alternate. The clostridia, particularly $C$. acetylbutyricum possess the ability to produce organic solvents such as acetone and butanol which can be modified and adapted to produce butyric acid. The optimum culture conditions include maintaining the $\mathrm{pH}$ of the medium above 5.0 in order to direct the fermentation away from solvent so that formation of butyric acid is favoured. ${ }^{2}$

Esters: Esters are generally added in food products like beverages, candies, jellies, jams, baked goods, wines, and dairy products such as cultured butter, sour cream, yogurt, and cheese. They contribute to a fruity aroma to the food. The derivatives of esters like acetate esters, such as ethyl acetate, hexyl acetate, isoamyl acetate and 2-phenylethyl acetate provide typical flavour in wine and other grape-derived alcoholic beverages. Several strains of lactic acid bacteria synthesize 
ethyl esters and thioesters. Lactococcus lactis possess unique esterase enzyme that is responsible for the formation of these aroma ester compounds. ${ }^{18}$ Several non-Saccharomyces wine yeasts are also the producers of acetate ester. ${ }^{19}$ The yeasts Hanseniaspora guilliermondii and Pichia anomala are potent 2-phenylethyl acetate and isoamyl acetate producers, respectively. In cheese production, ethyl or methyl esters of short-chain fatty acids contribute to the fruity flavour while thioesters derived from thiols are associated with cabbage or sulphur aromas. ${ }^{20}$

Isovaleric Acid: Isovaleric acid $\left.\left[\left(\mathrm{CH}_{3}\right)_{2} \mathrm{CHCH}_{2} \mathrm{CO}_{2} \mathrm{H}\right)\right]$ is responsible for acid-acrid odor commonly described as 'locker room 'or 'dirty feet'. This is one of the most offensive odors in the flavor and fragrance industry. It has a tenacious affinity for the skin. Isovaleric acid is generally used in very dilute concentrations to make its smell agreeable and herbaceous. It is widely used in its ester form in the flavor industry e.g. ethyl isovalerate possesses a powerful apple-fruity odor and used in numerous fruit flavors, candies and chewing gums. Besides isopentyl isovalerate has a fruit-apple-raspberry odor and is commonly used in apple flavors and as a modifier in numerous fruit and nut flavors. Another ester form isobutyl isovalerate possess appleraspberry-like note and is used in fruit flavors and in perfumes for lipsticks. $^{2}$

Pyrazines: Pyrazines are chemically heterocyclic, nitrogen-containing compounds that are normally produced during conventional cooking or roasting of food through the Maillard reaction. This class of flavour compounds is responsible for a nutty and roasted flavour in food. However, the microwave cooking does not favour pyrazine formation and so the natural pyrazines are externally added as food additives to provide a roasty flavour. There are few microorganisms that synthesize pyrazines, e.g. bacteria such as Corynebacterium glutamicum produce important quantities of tetramethylpyrazine from amino acids. ${ }^{2}$

Geosmin: Geosmin is responsible for imparting the earthy smell after the first rain. It was first isolated by scientists Gerber and Lechevalier and produced by blue-green algae, myxomycetes, actinomycetes and other microorganisms. The characteristic earthy odour of soil after rain is due to the release of geosmin by various Actinomycetes present in the soil. Geosmin is responsible for contaminating water supplies that result in unpalatable water, both for fish and animal drinking. Geosmin has two unique properties, firstly its odor is detectable in water at a concentration of $0.2 \mu \mathrm{g} / \mathrm{l}$, and secondly it has the ability to 'fatigue' the nose rapidly, making its organoleptic evaluation difficult. However, the earthy notes of geosmin are useful in imparting or modifying amber nots in perfume. Geosmin is used for the reconstitution of natural essential oils at a concentration of 1-100ppm. Geosmin can also be produced in fermenters using Streptomyces griseus LP-16 in a soybean meal-peptone-salt-glucose medium. ${ }^{2}$

Vanillin: Vanillin is a unique flavour chemical that occurs in Vanilla planifolia beans. Its chemical formula is 4-hydroxy-3methoxybenzaldehyde. This flavour is widely used in foods, beverages, perfumes, pharmaceuticals and in various medical industries. ${ }^{8}$ Although vanillin can be chemically synthesized, but there is an increasing demand for natural vanillin..$^{21}$ The direct extraction of vanillin from vanilla beans is expensive and limited which makes this compound a promising target for biotechnological flavour production. Vanillin is also produced as an intermediate compound in the microbial degradation of several substrates such as ferulic acid, phenolic stilbenes, lignin, eugenol and isoeugenol. Several bacterial and fungal strains of Pseudomonas putida, Aspergillus niger, Corynebacterium glutamicum, Corynebacterium sp., Arthrobacter globiformis and
Serratia marcescens are capable of conversion of natural eugenol and isoeugenol from essential oils into vanillin. ${ }^{8,22}$

Benzaldehyde: Benzaldehyde is naturally extracted from fruit kernels such as apricots and imparts a flavour similar to cherry. This extraction also leads to a formation of toxic hydrocyanic acid that is undesirable. An alternative method of extraction is microbial fermentation without producing any harmful by-products. However, benzaldehyde is toxic towards microbial metabolism and its accumulation in the culture medium inhibits cell growth. Thus, only a few microorganisms such as Pseudomonas putida and the white rot fungi Trametes suaveolens, Polyporus tuberaster, Bjerkandera adusta and Phanerochaete chrysosporium can be used as benzaldehyde producers. They are involved in the biosynthesis of benzaldehyde from phenylalanine. ${ }^{2}$

Terpenes: Terpenes are widespread in nature and mainly found in plants as constituents of essential oils. The common ones are linalool, nerol, geraniol and citronellol and they are most flavour-active due to their low sensory threshold. Research has shown that aromas and fragrances can also be produced through microbial oxidation of monoterpenes. Terpenes are usually produced by fungi belonging to group ascomycetes and basidiomycetes. The fungus Ceratocystis moniliformis produces several aroma products such as ethyl acetate, propyl acetate, isobutyl acetate, isoamyl acetate, citronellol and geraniol. Many microorganisms have an ability to break down terpenes and to carry out specific conversions producing value added products. Some of the enzymes involved in terpene biosynthesis have been sequenced, e.g., monoterpene synthase (isolated from sweet basil) is a key enzyme involved in the production of geraniol and has been sequenced to produce recombinant geraniol synthase. ${ }^{23}$ In addition, another geraniol synthase was cloned from the camphor tree Cinnamomum tenuipilum and was expressed in E. coli. ${ }^{24}$ Functional genomics was used to identify the genes for monoterpene synthases from Vitis vinifera grapes in order to characterize the enzymes by expression in E. coli and subsequent analysis. ${ }^{25}$

Flavour and fragrance production through microbial oxidation of monoterpenes: There are some microorganisms that can be metabolically modified to carry out specific conversions of substrates of low commercial value. Aspergillus niger IOC-3913 has an ability to produce flavor compounds through oxidation of terpene compounds. This species is highly resistant to toxic monoterpenic substrates. ${ }^{7}$

\section{Microbial production of aroma and fragrance through Solid State Fermentation (SSF)}

Solid state fermentation (SSF) is defined as any fermentation process performed on a non-soluble material that acts both as physical support and source of nutrients in absence of free flowing liquid. Solid state fermentation is better than submerged fermentation because its yields are much higher and gives better product characteristics. The cost of running SSF is much lower due to the efficient utilization and value-addition of wastes. ${ }^{26}$ Various studies has been carried out using SSF for the production of aroma compounds by several microorganisms, such as Neurospora sp., Zygosaccharomyces rouxii and Aspergillus sp., on variety of solid substrates like pre-gelatinized rice, miso and cellulose fibres, respectively. ${ }^{2}$ Special fruity aroma can be produced using Ceratocystis fimbriata in solid-state cultures using several agro-horticultural wastes like cassava bagasse, apple pomace, amaranth and soybean. A strong pineapple aroma can be produced by Ceratocystis fimbriata on coffee husk when used as a substrate in $\mathrm{SSF}^{27}$ Major flavour compounds identified were acetaldehyde, ethanol, ethyl acetate, ethyl isobutyrate. A strong odour of banana can 
be produced by adding leucine in the substrate for SSF that further enhanced the production of ethyl acetate and isoamyl acetate. De Araujo et al., ${ }^{28}$ studied the production of 6-pentyl-a-pyrone (6-PP), an unsaturated lactone with a strong coconut-like aroma, using both liquid and solid substrates in SSF process. Sugarcane bagasse was used as a substrate for growth and aroma production. Semisolid maize-based culture can be used to produce a typical dairy flavour compounds like diacetyl, butyric acid and lactic acid by using mixed cultures of Lactobacillus acidophilus and Pediococcus pentosaceus. ${ }^{29}$

As discussed above, the alternative methods to synthesize flavour and fragrance chemicals by biotechnological routes include mainly microbial fermentation and plant tissue culture. Microorganisms during fermentation also produce a vast array of aroma compounds. These aroma chemicals supplement and enhance the quality of plant based flavour and fragrance chemicals. Some of the recent developments of commercialized process to produce and bio-transform natural precursors into valuable flavour and fragrance chemicals through microbial metabolic pathways includes the production of tuberose lactone (a new GRAS chemical) through hydroxylation of unsaturated fatty acids and limited $\beta$-oxidation of the hydroxylated fatty acids; production of chirally active (R)-styrallyl acetate by regioselective reduction of acetophenone to styrallyl alcohol and subsequent esterification; and de novo synthesis of chirally pure $(+)$-jasmonic acid and subsequent esterification to methyl jasmonate. Microbial biotransformation and biosynthesis of flavor and fragrance chemicals offer the potential benefits of producing optically active isomers which often have marked differences in flavor and fragrance quality and sensory intensity. ${ }^{9}$

\section{Conclusion}

Currently most of the aroma compounds are extracted from synthetic sources or from natural sources of plants. But recent advances in metabolic engineering has generated a great interest for natural products particularly the aroma industry to seek new methods to obtain fragrance and flavour compounds naturally. An alternative and attractive route for fragrance and flavour compounds is based on microbial biosynthesis and bio-transformations. The above study indicates that microorganisms can be used as a safe player for the production of bio-flavours and fragrances. These microbes can be metabolically and genetically modified to enhance the production of desired metabolite of significance. Moreover, the production of aroma compounds from microbial cultures or their enzyme preparations offers several advantages over traditional methods. The microbial metabolites can be produced in large quantities by the use of solidstate fermentation and can give high quality yields with better product characteristics along with low economical costs.

\section{Acknowledgments}

Authors are grateful to Dr. Ashok K. Chauhan, Founder President, Amity Group of Institutions and Mr. Atul Chauhan, Chancellor, Amity University UP, Noida for the encouragement, research facilities and financial support.

\section{Conflicts of interest}

Authors declare that there is no conflict of interest.

\section{References}

1. Bomgardner MM. The Sweet Smell of Microbes. Chemical and Engineering News. 2012;90(29):25-29.
2. Longo MA, Sanroman MA. Production of Food Aroma Compounds. Food Technol. Biotechnol. 2006;44(3):335-353.

3. Romero-Guido C, Belo I, Ta TM, et al. Biochemistry of lactone formation in yeast and fungi and its utilization for the production of flavour and fragrance compounds. Appl Microbiol Biotechnol. 2011;89(3):535-547.

4. Richmond B. How to Make Perfume Out of Bacteria Instead of Oil. Motherboard; 2014.

5. Biotechnology and Biological Sciences Research Council. Plant perfumes woo beneficial bugs to their roots. Science Daily; 2014.

6. Wackett LP. Microbiology for odour production and abatement. Microbial Biotechnology. 2012;6(1):85-86.

7. Rozenbaum HF, Patitucci ML, Antunes OAC, et al. Production of aromas and fragrances through microbial oxidation of monoterpenes. Brazilian Journal of Chemical Engineering. 2006;23(3):273-279.

8. Priefert H, Babenhorst J, Steinbuchel A. Biotechnological production of vanillin. Appl Microbiol Biotechnol. 2001;56(3-4):296-314.

9. Kim AY. Application of Biotechnology to the Production of Natural Flavor and Fragrance Chemicals. Natural Flavors and Fragrances ACS Symposium Series (Chapter 4). 2005;908:60-75.

10. Krings U, Berger RG. Biotechnological production of flavours and fragrances. Appl Microbiol Biotechnol. 1998;49(1):1-8.

11. Wiley B. Bacteria Manage Perfume Oil Production From Grass. Science Daily; 2008.

12. Natural health care news. URL http://naturalhealthcare.ca/news. phtml?id=3029\&sac $=$ view

13. Neal AL, Ahmad S, Gordon-Weeks R, et al. Benzoxazinoids in root exudates of maize attract Pseudomonas putida to the rhizosphere. PLoS One. 2012;7(4):e35498.

14. Mallouchos A, Komaitis M, Koutinas A, et al. Wine fermentations by immobilized and free cells at different temperatures. Effect of immobilization and temperature on volatile by-products. Food Chemistry. 2003;80(1):109-113.

15. Nomura K, Ogura H, Imanishi Y. Direct synthesis of 2-phenylethanol by hydrogenation of methyl phenyl acetate using homogeneous rutheniumphosphine catalysis under low hydrogen pressure. Journal of Molecular Catalysis A: Chemical. 2001;166(2):345-349.

16. Stark D, Munch T, Sonnleitner B, et al. Extractive bioconversion of 2-phenylethanol from L-phenylalanine by Saccharomyces cerevisiae. Biotechnol Prog. 2002;18(3):514-523.

17. Starka D, Zalaa D, Munchb T, et al. Inhibition aspects of the bioconversion of L-phenylalanine to 2-phenylethanol by Saccharomyces cerevisiae. Enzyme and Microbial Technology. 2003;32(2):212-223.

18. Nardi M, Fiez-Vandal C, Tailliez P, et al. The EstA esterase is responsible for the main capacity of Lactococcus lactis to synthesize short chain fatty acid esters in vitro. J Appl Microbiol. 2002;93(6):994-1002.

19. Rojas V, Gil JV, Pinaga F, et al. Studies on acetate ester production by non-Saccharomyces wine yeasts. International Journal of Food Microbiology. 2001;70(3):283-289.

20. Liu SQ, Holland R, Crow VL. Esters and their biosynthesis in fermented dairy products: A review. International Dairy Journal. 2004;14(11):923-945.

21. Walton NJ, Narbad A, Faulds C, et al. Novel approaches to biosynthesis of vanillin. Curr Opin Biotechnol. 2000;11(5):490-496.

22. Shimoni E, Baasov T, Ravid U, et al. Biotransformations of propenylbenzenes by an Arthrobacter sp. and its t-anethole blocked mutants. J Biotechnol. 2003;105(1-2):61-70. 
23. Pichersky E, Iijima Y, Lewinsohn E, et al. Cloning and sequence of geraniol synthase from sweet basil and use in the production of geraniol and metabolites. International patent PCT WO 2005060553; 2005.

24. Yang T, Li J, Wang HX, et al. A geraniol-synthase gene from Cinnamomum tenuipilum. Phytochemistry. 2005;66(3):285-293.

25. Martin DM, Bohlmann J. Identification of Vitis vinifera (-)-alphaterpineol synthase by in silico screening of full-length cDNA ESTs and functional characterization of recombinant terpene synthase. Phytochemistry. 2004;65(9):1223-1229.

26. Robinson T, Nigam P. Bioreactor design for protein enrichment of agricultural residues by solid state fermentation. Biochemical Engineering Journal. 2003;13(2-3):197-203.
27. Soares M, Christen P, Pandey A, et al. Fruity flavour production by Ceratocystis fimbriata grown on coffee husk in solid state fermentation. Process Biochemistry. 2000;35(8):857-861.

28. De Alberto AA, Pastore GM, Berger RG. Production of coconut aroma by fungi cultivation in solid-state fermentation. Appl Biochem Biotechnol. 2002;98-100:747-751.

29. Escamilla-Hurtado ML, Valdes-Martinez SE, Soriano-Santos J, et al. Effect of culture conditions on production of butter flavor compounds by Pediococcus pentosaceus and Lactobacillus acidophilus in semisolid maize-based cultures. Int J Food Microbiol. 2005;105(3):305-316. 